\title{
KRIGAGEM E INVERSO DO QUADRADO DA DISTÂNCIA PARA INTE R POLAÇÃO DOS PARÂMETROS DA EQUAÇÃO DE CHUVAS INTENSAS ${ }^{(1)}$
}

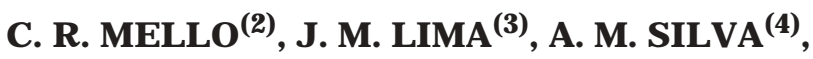 \\ J. M. MELLO(5) \& M.S. OLIVEIRA ${ }^{(6)}$
}

\begin{abstract}
RESUMO
E m projetos de obras hidráulicas, é necessária a esti mativa da chuva intensa adequada à realidade técnico-financeira dos projetos, para posterior cálculo de vazões máximas. O uso da equação de chuvas intensas é a forma mais usual para estimativa da chuva de projeto. No entanto, há dificuldade em obtêla para locais desprovidos de dados pluviométricos, o que pode ser superado mediante técnicas computacionais para a espacialização dos seus parâmetros. Para isto, pode-se trabalhar com procedimentos estatísticos para interpolação dos parâmetros com base em estações meteorológicas vizinhas ao local. Objetivouse, neste trabalho, comparar os dois procedimentos estatísticos mais exatos, conforme já constatado em outras aplicações físicas, que são a krigagem (interpolador geoestatístico) e o inverso do quadrado da distância, para interpolação dos parâmetros da equação de chuvas intensas. Utilizaram-se 140 estações meteorológicas do estado de São Paulo, as quais dispõem da respectiva equação de chuvas intensas estimada com base em dados pluviométricos, consideradas como ideais. Destas, 126 foram utilizadas para estudo da dependência espacial dos parâmetros e 14 para comparação de erros nas estimativas da chuva intensa. Constatou-se que ambos os métodos apresentaram boa precisão, mas a krigagem produziu menores erros médios para 11 estações, verificando-se melhorias consideráveis em especial para tempos de retorno e tempos de duração da preci pitação usuais em projetos em pequenas bacias hidrográficas, podendo-se sugerir este método como o mais adequado.
\end{abstract}

Termos de indexação: chuva intensa, espacialização, geoestatística, hidrologia.

(1) Trabal ho financiado pela FAPE MIG. Recebido para publicação em novembro de 2002 e aprovado em julho de 2003.

(2) Doutorando do Departamento de Ciência do Solo, Universidade Federal de Lavras - UFLA. Caixa Postal 37, CEP 37200-000 Lavras (MG). E-mail: crmello@ufla.br.

(3) Professor do Departamento de Ciência do Solo, UFLA. Bolsista do CNPq. E-mail: jmlima@ufla.br

(4) Professor do Departamento de Engenharia, UFLA. Bolsista do CNPq. E-mail: marciano@ufla.br

(5) Professor do Departamento de Ciências Florestais, UFLA. E-mail: jmmello@esalq.usp.br

(6) Professor do Departamento de Ciências Exatas, UFLA. E-mail: marcelso@ufla.br 


\title{
SUMMARY: KRIGING AND INVERSE-SQUARE-DISTANCE FOR THE INTERPOLATION OF RAINFALL EQUATION PARAMETERS
}

\begin{abstract}
To design hydraulic structures, a sound estimati on of rainfall parameters is necessary, in order to ensure the technical and economical feasibility of the project. Equations to predict intenserainfall are widel y used to esti mate runoff peak discharge Thedifficulty to obtain estimates for sites where no rainfall data are available can be overcome by the interpolation of precipitation data, based on neighboring meteorol ogical stations, through statistical procedures. The objective of this work was to compare the two most accurate statistical methods, previously confirmed in other applications, to interpolate rainfall parameters: kriging (geostatistical interpolator) and the inversesquaredistance, for the interpolation of equation parameters of intense rainfall. Data from 140 meteorological stations in Sao Paulo State, Brazil, were used. Each station has its estimated equation of intenserainfall, which is considered ideal, based on historical data series. Spatial dependence of the parameters was tested for 126 of the stations, whereas the other 14 were used to compare the mean errors of the two methods. Both allowed a satisfactory estimation of rainfall parameters. Thekriging method can beindicated as the most appropriate sinceit produced lower mean errors at 11 stations, especially for the return period and duration time commonly applied to esti mate rainfall parameters for projects in small watersheds.
\end{abstract}

Index terms: intense rainfall, regionalization, geostatistics, hydrol ogy.

\section{INTRODUÇÃO}

O conhecimento da chuva de áreas do projeto é essencial para o êxito de um projeto de engenharia ligado a obras hidráulicas. Para o bom dimensionamento de barragens para contenção do excesso de água e, ou, represamento para fins múltiplos, terraços e bacias de contenção de erosão, drenagem rural e urbana, pontes, canais e outras estruturas hidráulicas, a estimativa mais precisa possível de uma chuva intensa é extremamente importante para que um projeto seja viável tanto do ponto de vista técnico quanto econômico.

A utilização de equações de chuvas intensas é a forma mais usual para estimar uma intensidade de chuva a ser aplicada na determinação de vazões de cheia (Tucci, 1998; Chow et al., 1988; Genovez, 2001). Para estimar os parâmetros de chuvas intensas, trabalha-se com regressão múltipla não-linear utilizando-se séries históricas de dados de chuvas máximas com vários tempos de duração, extraídos de pluviogramas cotados. Quando não se dispõe deste instrumento, pode-se trabalhar com dados pluviométricos de chuvas máximas diárias anuais aplicando-se o processo de desagregação de chuvas, que é bastante comum (M ello et al., 2001; Cardoso et al., 1998).

Na ausência deinformações, tanto pluviométricas quanto pluviográficas, Silva et al. (1999), Pinto (1999) e Assad et al. (1994) sugerem trabal har com métodos que determi nam uma média ponderada dos parâmetros da equação de chuvas intensas pelo inverso da distância, geralmente com o quadrado da distância, obtendo-se, desta forma, uma equação dechuvas intensas para locais desprovidos de dados pluviométricos, utilizando dados de estações meteorológicas adjacentes. Este método tem sido empregado e sugerido atualmente, conforme os trabalhos citados, em especial os dois primeiros.

Myers (1994) e Laslett (1994) fizeram uma abordagem geral de métodos utilizados para interpolação espacial de parâmetros em áreas de pesquisa como ciência do solo, ecologia, engenharia florestal, hidrologia e meteorologia, destacando-se a krigagem (ou interpol ação geoestatística) como um preditor em potencial de inúmeros parâmetros nestas áreas. Dirks et al. (1998) trabalharam com al guns interpoladores espaciais para chuvas anuais, mensais, diárias e horárias para a Il ha de Norfolk na Nova Zelândia. Os pesquisadores concluíram que os métodos krigagem e inverso do quadrado da distância sobressaíram em relação a polígonos de Thiessen, splines cúbicas e inverso da distância com expoentes 1, 3 e 4 . Contudo, os autores não estabel eceram qual dos dois métodos citados poderia produzir maior precisão.

Trabalhando com atributos de solo, Voltz \& Webster (1990) e Gotway et al. (1996) concluíram que a krigagem proporcionou os melhores resultados, quando comparada, respectivamente, a splines cúbicas e métodos de inverso da distância, dentre eles o de expoente 2.

Segundo Vieira (2000) e Thompson (1992), a krigagem tem capacidade de produzir melhores estimativas em termos de interpolação, porque está embasada em duas premissas: não-tendenciosidade 
do estimador e variância mínima das estimativas. Além disto, quando se trabalha considerando a dependência espacial, podem-se reduzir os erros aleatórios pelo controle de parcela deste erro associada à dependência espacial. Deve-seressaltar que a krigagem mostra uma ótima qualidade estatística explícita, exatamente as propriedades descritas anteriormente, enquanto o inverso do quadrado da distância não tem tal qualidade objetiva. A única baseteórica para adoção do inverso do quadrado da distância talvez seja sua semelhança coma Lei de Gravitação Universal eLei de Coulomb (Força eCampoE létrico), ambas com enfraquecimento diretamente proporcional ao inverso da distância.

Em termos devariáveis hidrológicas, em especial chuvas máximas diárias, Vieira et al. (1991) trabalharam com 232 estações meteorológicas do estado de São Paulo, empregando interpolação geoestatística, graças à estrutura de dependência espacial detectada para esta variável hidrológica. Como os parâmetros da equação de chuvas intensas são estimados com base em dados de chuvas máximas diárias, acredita-se haver estrutura de dependência espacial dos dados. Assim, a krigagem pode permitir uma melhor estimativa destas que outros métodos de interpolação, inclusive o inverso do quadrado da distância, que vem sendo proposto.

Os objetivos deste trabal ho são: (a) determinar a existência de dependência espacial dos parâmetros da equação de chuvas intensas; (b) no caso de dependência espacial, comparar as interpolações pelos métodos: krigagem geoestatística einverso do quadrado da distância.

\section{MATERIAL E MÉTODOS}

A equação de chuvas intensas apresenta a seguinte forma geral (Righetto, 1998):

$$
\mathrm{I}=\frac{\mathrm{K} \cdot \mathrm{TR}^{\mathrm{a}}}{(\mathrm{td}+\mathrm{B})^{\mathrm{C}}}
$$

em que I: intensidade da precipitação $\left(L T^{-1}\right)$; TR: tempo de retorno (anos); td: tempo de duração da chuva (T); K, a, B e C: parâmetros de ajuste, adimensional. Trabalhando com dados de precipitação máxima em diferentes tempos de duração, constituindo-se séries históricas, ajustamse os parâmetros citados por meio de regressão múltipla não-linear (Haan, 1979; Froehlich, 1993).

F oram empregadas informações de equações de chuvas intensas e respectiva localização geográfica de 140 estações meteorológicas do estado de São Paulo, ajustadas com base em série histórica de dados pluviográficos, por meio do programa Plúvio 1.3, desenvolvido pelo Departamento de Engenharia Agrícola da Universidade Federal de
Viçosa, o qual foi baseado em trabalho de Silva et al. (1999) e Pinto (1999).

Destas 140 estações, 126 foram empregadas para o estudo de dependência espacial dos parâmetros por meio de semivariograma experimental isotrópico a partir da expressão (J ournel, 1989):

$$
\gamma^{*}(\mathrm{~h})=\frac{1}{2 \mathrm{~N}(\mathrm{~h})} \cdot \sum_{\mathrm{i}=1}^{\mathrm{n}(\mathrm{h})}[\mathrm{Z}(\mathrm{s}+\mathrm{h})-\mathrm{Z}(\mathrm{s})]^{2}
$$

em que $Z(s)$ e $Z(s+h)$ são pares de valores medidos em locais afastados entre si da distância h e N(h), o número desses pares para cada h. O uso desse estimador pressupõe que a propriedade atenda a uma condição de estacionaridade, segundo a qual, no mínimo a hipótese intrínseca éatendida (J ournel \& Huijbregts, 1978).

Ao semivariograma experimental foram ajustados o model o exponencial $(K, B$ e $C$ ) e esférico (a), respectivamente definidos por McBratney \& Webster (1986), como se segue:

$$
\gamma(\mathrm{h})=\mathrm{C}_{\mathrm{o}}+\mathrm{C}_{1} \cdot\left[1-\exp \left(\frac{-3 \cdot \mathrm{h}}{\mathrm{a}}\right)\right] \text { para } 0<\mathrm{h}<\mathrm{a}
$$

e

$$
\gamma(\mathrm{h})=\mathrm{C}_{\mathrm{o}}+\mathrm{C}_{1} \cdot\left[1,5 \cdot\left(\frac{\mathrm{h}}{\mathrm{a}}\right)-0,5 \cdot\left(\frac{\mathrm{h}}{\mathrm{a}}\right)^{3}\right] \text { para } 0<\mathrm{h}<\mathrm{a}
$$

sendo $\gamma(\mathrm{h})$ : semivariância; h: distância máxima em que o semivariograma é definido; $C_{0}$ : efeito pepita (ponto de interseção da curva com o eixo y); $\mathrm{C}_{1}$ : patamar - $\mathrm{C}_{0}$; a: alcance do semivariograma (distância na qual se detecta dependência espacial). Estes model os foram ajustados por meio do programa Variowin, desenvolvido por Pannatier (1994).

O interpolador geoestatístico, conhecido como krigagem, apresenta a seguinte seqüência de cálculos, segundo Thompson (1992):

$$
[\mathrm{A}]^{-1} \cdot[\mathrm{b}]=[\lambda]
$$

$[\mathrm{A}]^{-1}$ : matriz inversa de semivariância entre as localidades da vizinhança de um ponto, determinada pelo modelo de semivariograma com base nas distâncias euclidianas entre as localidades; [b]: matriz de semivariância entre as localidades vizinhas (com a variável estimada) e o ponto para o qual a variável será interpolada, também determinado pelo modelo de semivariograma, com base nas distâncias entre as localidades vizinhas e o ponto a ser interpolado; $[\lambda]$ : matriz de pesos de krigagem.

$$
X_{p}=\sum_{i=1}^{n} \lambda_{i} \cdot X_{i}
$$


$\mathrm{X}_{\mathrm{p}}$ : variável interpolada; $\lambda_{1}$ : peso da i-ésima localidade vizinha; $\mathrm{X}_{\mathrm{i}}$ : valor da variável para a $\mathrm{i}$ ésima local idade; n: número delocal idades vizinhas empregadas para interpolação do ponto.

A interpolação por meio do inverso do quadrado da distância foi feita da seguinte forma:

$$
X_{p}=\frac{\sum_{i=1}^{n}\left(\frac{1}{d_{i}{ }^{2}} \cdot X_{i}\right)}{\sum_{i=1}^{n}\left(\frac{1}{d_{i}{ }^{2}}\right)}
$$

Xp: variável interpolada; $X_{i}$ : valor da variável da iésima localidade vizinha; $d_{i}$ : distância euclidiana entre o i-ésimo ponto de vizinhança e o ponto amostrado.

No presente estudo, trabalhou-se com as 6 estações mais próximas da localidade a ser interpolada, conforme método adotado por Silva et al. (1999), levando em consideração apenas as local idades que estivessem dentro do rai o do al cance espacial obtido por cada parâmetro.

Para análise de erros, foram utilizadas as 14 estações meteorológi cas restantes (das 140 iniciais) com os valores dos parâmetros da equação de chuvas intensas estimados com base em série histórica de dados pluviográficos. Assim, foi possível comparar os métodos, calculando-se as chuvas intensas com base nas equações ajustadas pel as interpolações com os valores determinados pelos parâmetros reais. Desta forma, foram estimadas chuvas intensas para o tempo de retorno de 5, 10, 15, 20, 50, 75 e 100 anos e tempos de duração de 10, 30, 60, 120, 240, 360, $720,1.440,2.160$ e 2.880 minutos (48 horas). Os erros foram calculados da seguinte forma, segundo Dirks et al. (1998) e Mello et al. (2001):

$$
\begin{gathered}
\mathrm{e}=\frac{\left|\mathrm{V}_{\mathrm{R}}-\mathrm{V}_{\mathrm{P}}\right|}{\mathrm{V}_{\mathrm{P}}} \cdot 100 \\
\mathrm{e}_{\mathrm{m}}=\frac{\sum_{\mathrm{i}=1}^{\mathrm{n}} \mathrm{e}_{\mathrm{i}}}{\mathrm{n}}
\end{gathered}
$$

em que $V_{R}$ : valor da variável real; $V_{p}$ : valor da variável predita; e: erro da i-ésima observação (\%); e $e_{m}$ erro médio (\%); n: número de situações avaliadas (os 10 tempos de duração e 7 tempos de retorno, listados anteriormente, total izando 70 observações).

Objetivando também determinar sobre quais condições de tempo de duração e tempo de retorno um método pode ser mais exato que outro, foi feita uma abordagem comparativa mais completa do comportamento dos erros proporcionados pelos métodos, considerando os intervalos destacados anteriormente para estas variáveis (tempo de duração e tempo de retorno).

\section{RESULTADOS E DISCUSSÃO}

\section{Avaliação da dependência espacial dos parâmetros da equação de chuvas intensas}

No quadro 1, são apresentados os val ores médios e os coeficientes de variação (CV) para os quatro parâmetros da equação de chuvas intensas. Observa-se que os parâmetros K e B apresentaram el evados CV, acima de $30 \%$, mostrando alta variabilidade. Para os parâmetros a e C, os coeficientes de variação encontrados foram consideravel mente menores, sendo de 16,1 e 10,9\%, respectivamente. Vieira et al. (1991) encontraram coeficientes de variação semel hantes para o estado de São Paulo, estudando a dependência espacial de chuvas máximas diárias, mostrandoa possibilidade de haver estrutura de dependência espacial destes parâmetros.

Na figura 1, são mostrados os semivariogramas ajustados para cada um dos parâmetros e, na seqüência, os respectivos modelos.

$$
\begin{array}{r}
\gamma(\mathrm{h})=140.000+120.000 \cdot\left[1-\exp \left(\frac{-3 \cdot \mathrm{h}}{93.074}\right)\right] \\
\gamma(\mathrm{h})=15,37+29,15 \cdot\left[1-\exp \left(\frac{-3 \cdot \mathrm{h}}{92.400}\right)\right] \\
\gamma(\mathrm{h})=0,004+0,0034 \cdot\left[1-\exp \left(\frac{-3 \cdot \mathrm{h}}{92.400}\right)\right] \\
\gamma(\mathrm{h})=0,0006 \cdot\left[1,5 \cdot\left(\frac{\mathrm{h}}{103.600}\right)-0,5 \cdot\left(\frac{\mathrm{h}}{103.600}\right)^{3}\right]
\end{array}
$$

Observa-sequeos parâmetros K eB apresentaram os melhores ajustes, com um patamar elevado, garantindo boa dependência espacial dos citados parâmetros, especialmente o parâmetro $B$, que, além disto, apresenta um baixo efeito pepita. Os parâmetros $\mathrm{C}$ e a também apresentaram bons ajustes dos respectivos modelos, com baixos efeitos pepita e patamares suficientemente el evados para produzir uma boa estrutura de dependência espacial.

\begin{tabular}{ccc}
$\begin{array}{r}\text { Quadro 1. Valores médios e coeficientes de variação } \\
\text { dos parâmetros da equação de chuvas intensas } \\
\text { para as } \mathbf{1 2 6} \text { estações meteorológicas avaliadas }\end{array}$ \\
\hline Parâmetro & CV & Média (adimensional) \\
\hline & $\%$ & \\
K & 32,9 & 1520,328 \\
a & 16,1 & 0,136 \\
B & 32,3 & 19,048 \\
C & 10,9 & 0,793 \\
\hline
\end{tabular}




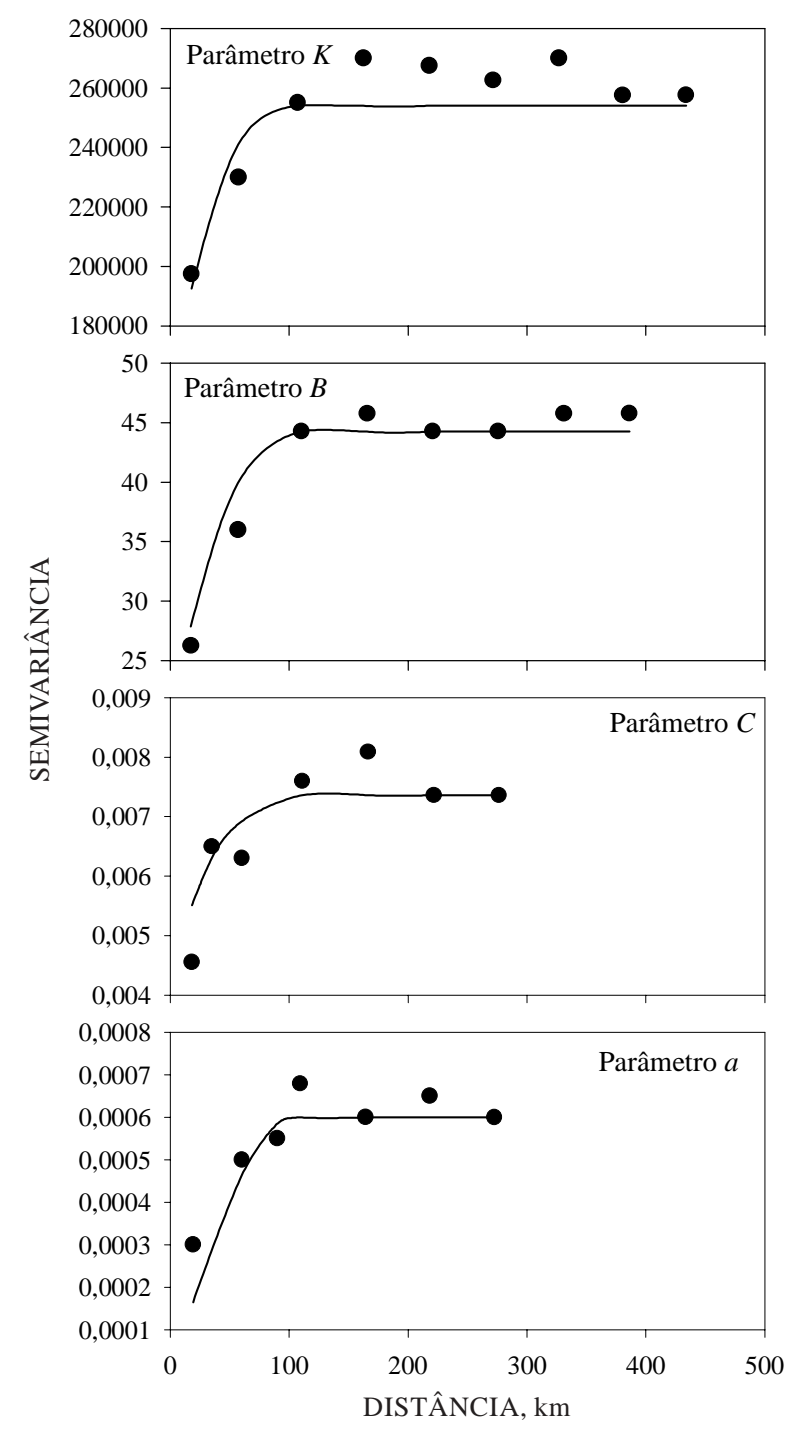

Figura 1. Semivariogramas dos parâmetros K, B, C e a da equação de chuvas intensas.
Desta forma, o uso do interpolador geoestatístico pode perfeitamente ser aplicado. Pode-se observar também que os alcances encontrados ficaram próximos uns dos outros, na faixa de 90 a $100 \mathrm{~km}$. Vieira et al. (1991) também trabalharam com o model o exponencial para chuvas máximas diárias, concluindo o melhor ajuste deste modelo, e encontraram alcance para esta variável hidrológica próximoa $100 \mathrm{~km}$. Esta situação reforça ainda mais a estrutura de dependência espacial que há para os parâmetros estudados, haja vista a forte relação entre eles e a chuva máxima diária.

\section{Avaliação dos erros produzidos pelos métodos de interpolação}

No quadro 2, são mostrados os val ores interpolados pelos métodos inverso do quadrado da distância e krigagem para os quatro parâmetros da equação de chuvas intensas, bem como o valor estimado com base em séries históricas de precipitação máxima diária, para efeito de comparação. A análise individual dos parâmetros não tem significado físico, uma vez que a aplicação da equação de chuvas intensas é uma combinação de todos os parâmetros, inter essando, portanto, a chuva intensa gerada pela equação como um todo. Além disso, determinado parâmetro podeser interpolado com maior precisão por um dos métodos, enquanto outro parâmetro interpolado podeser mais bem interpolado por outro método. Esta combinação de precisões influi na estimativa final da chuva intensa.

No entanto, algumas observações importantes podem ser feitas. Pode-seobservar quea interpol ação do parâmetro a não apresenta grandes diferenças entre os métodos como as constatadas pel os outros parâmetros, em especial K e B. I sto está associado ao fato de que a estrutura de dependência espacial do parâmetro a não apresenta as mesmas qualidades produzidas pel os ajustes dos demais parâmetros.

Quadro 2. Valores interpolados pelos métodos estudados ( $K_{I Q D}$ e $K_{k r i g, ~} a_{I Q D}$ e $a_{K \text { rig.' }} B_{I Q D}$ e $B_{\text {krig, }} C_{I Q D}$ e $C_{\text {krig }}$ ) e medidos ( $K_{\text {Real }}, a_{\text {Real }}, B_{\text {Real }}$ e $C_{\text {Real }}$ ) para os parâmetros de equações de chuvas intensas para as 14 estações do estado de São Paulo utilizadas para o estudo de erros

\begin{tabular}{|c|c|c|c|c|c|c|c|c|c|c|c|c|}
\hline Cidade & KIQD & Kkrig. & $K_{\text {Real }}$ & QIQD & akrig. & areal & B IQD $_{\text {IQ }}$ & B Krig. & $B_{\text {Real }}$ & CIQD & C Krig. & Creal \\
\hline Boituva & 1609,9 & 1569,2 & 1999,6 & 0,131 & 0,128 & 0,186 & 20,56 & 20,34 & 17,79 & 0,819 & 0,810 & 0,881 \\
\hline Botucatu & 1403,1 & 1401,0 & 1405,8 & 0,119 & 0,134 & 0,139 & 23,04 & 22,50 & 18,37 & 0,752 & 0,756 & 0,787 \\
\hline Caçapava & 1188,7 & 1277,7 & 2000,0 & 0,134 & 0,135 & 0,115 & 10,04 & 9,46 & 24,99 & 0,799 & 0,801 & 0,852 \\
\hline Cubatão & 776,6 & 769,7 & 695,2 & 0,153 & 0,140 & 0,126 & 22,90 & 18,56 & 8,73 & 0,639 & 0,633 & 0,607 \\
\hline Dourado & 1800,4 & 1815,7 & 1999,7 & 0,122 & 0,126 & 0,149 & 21,98 & 22,35 & 22,48 & 0,835 & 0,836 & 0,849 \\
\hline Guarujá & 1112,3 & 1123,0 & 1349,3 & 0,135 & 0,130 & 0,155 & 18,84 & 20,23 & 23,95 & 0,696 & 0,695 & 0,757 \\
\hline Itanhaém & 1467,6 & 1457,6 & 1733,3 & 0,123 & 0,114 & 0,124 & 17,41 & 17,50 & 25,00 & 0,786 & 0,783 & 0,816 \\
\hline Itaporanga & 1220,9 & 1208,4 & 500,02 & 0,139 & 0,132 & 0,155 & 18,06 & 18,88 & 16,91 & 0,720 & 0,701 & 0,525 \\
\hline Itirapina & 1928,8 & 1898,7 & 1999,6 & 0,169 & 0,175 & 0,152 & 13,93 & 15,39 & 24,34 & 0,877 & 0,867 & 0,850 \\
\hline Lins & 1385,2 & 1429,3 & 2000,0 & 0,131 & 0,131 & 0,108 & 20,38 & 20,41 & 21,02 & 0,752 & 0,762 & 0,860 \\
\hline Martinópolis & 1409,9 & 1410,1 & 2000,0 & 0,132 & 0,132 & 0,136 & 23,41 & 23,16 & 24,66 & 0,760 & 0,765 & 0,856 \\
\hline Paranapanema & 1572,5 & 1661,9 & 1232,7 & 0,126 & 0,134 & 0,130 & 19,16 & 19,79 & 16,87 & 0,799 & 0,817 & 0,768 \\
\hline Pedro Toledo & 1520,4 & 1348,0 & 1215,6 & 0,136 & 0,129 & 0,166 & 21,03 & 20,17 & 25,00 & 0,776 & 0,737 & 0,709 \\
\hline Piacatu & 1390,8 & 1347,5 & 1995,4 & 0,141 & 0,135 & 0,099 & 20,98 & 20,96 & 25,00 & 0,758 & 0,753 & 0,818 \\
\hline
\end{tabular}

IQD = Inverso do Quadrado da Distância; Krig.=Krigagem. 
No quadro 3, encontram-se as 14 estações meteorológicas testadas com suas respectivas vizinhanças e os erros médios gerados para chuvas intensas pelos métodos de interpolação. Observase que, para as estações de Lins, I taporanga e Botucatu 2, os erros médios foram el evados, acima de $23 \%$, proporcionados por ambos os métodos, em especial para Lins, com a krigagem apresentando o menor valor para as duas primeiras estações. Para as demais, os erros proporcionados pelos métodos foram baixos o suficiente para avaliar que ambos os métodos apresentam boa exatidão. Deve-seressaltar que a krigagem promoveu menores erros em 11 das 14 estações avaliadas e, em al guns casos, como Pedro deTol edo, a melhoria na interpolação foi substancial. Dirks et al. (1998) encontraram erros médios na interpolação de precipitações diárias de até $77 \%$ para o inverso do quadrado da distância e 73 \% para a krigagem. Isto mostra que os erros encontrados na estimativa de chuvas intensas pelo presente trabal ho estão dentro da margem de aceitação.

Na figura 2, destacam-se a relação entre erros de estimativa da chuva intensa e os tempos de duração e retorno das chuvas proporcionados pelos métodos de interpolação para as estações climatológicas de Botucatu 2, Boituva e Paranapanema. Apesar de ser o erro médio proporcionado pelo IQD menor que o proporcionado pela krigagem, há situações em que esta proporciona erros menores.

Para Boituva, os erros aumentam com o aumento do tempo de retorno para durações da precipitação até $100 \mathrm{~min}$, independentemente do método. Para tempos de retorno superiores a cinco anos, este comportamento se estende até próximo a 1.000 min de duração da precipitação. A krigagem proporciona erros menores que o IQD para tempos de duração da precipitação inferiores a $100 \mathrm{~min}$ e tempo de retorno de cinco anos. Tal comportamento ocorre para tempos de duração cada vez maiores, à medida que o tempo de retorno aumenta. Considerando tempos de duração e de retorno normalmente utilizados em projetos em pequenas bacias rurais, a krigagem foi o método que proporcionou os menores erros de interpolação.

Apesar de os erros médios serem superiores, o comportamento dos erros para a estação cl imatológica de Botucatu 2 foi semel hanteao de Boituva; valendo

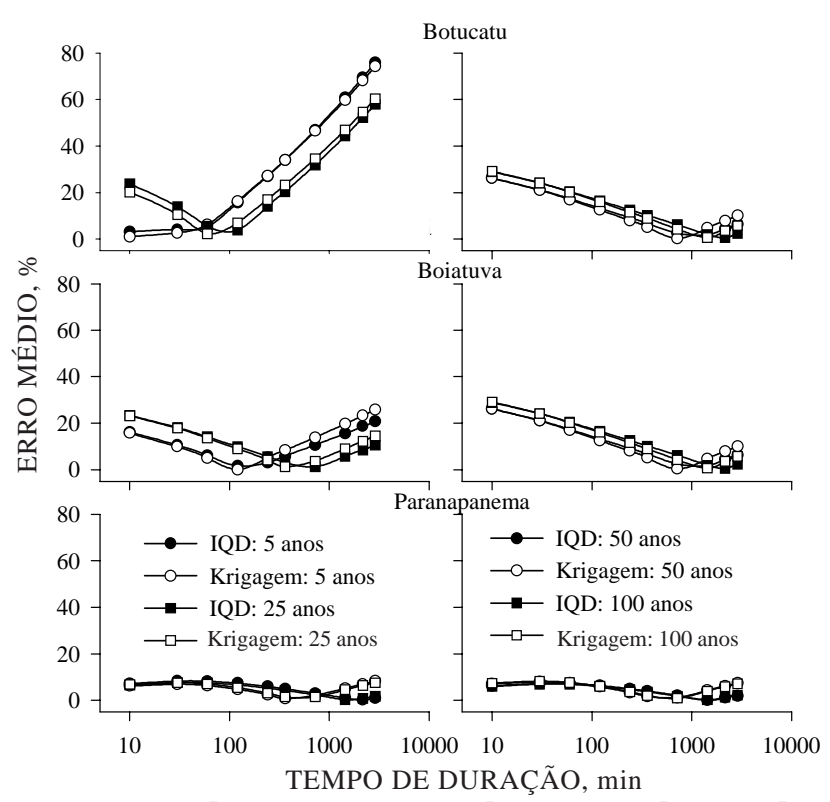

Figura 2. Relação entre erro das interpolações dos parâmetros de equações de chuvas intensas por krigagem e IQD com tempos de duração e de retorno para as localidades de Botucatu, Boituva e Paranapanema.

Quadro 3. Estações meteorológicas (coluna Cidade) testadas, bem como as vizinhanças empregadas em cada uma delas, e erros médios proporcionados pelos métodos de interpolação

\begin{tabular}{|c|c|c|c|c|c|c|c|c|}
\hline Cidade & Estação 1 & Estação 2 & Estação 3 & Estação 4 & Estação 5 & Estação 6 & $E_{\text {IQD }}$ & $E_{\text {Krig }}$ \\
\hline & & & & & & & \multicolumn{2}{|c|}{ — $\%$} \\
\hline Boituva & Itú & Elias Fausto & Piracicaba & Vinhedo & Tapiraí & Cosmópolis & 10,5 & 11,5 \\
\hline Botucatu 2 & Botucatu 1 & Itatinga & Santa M. Serra & Guaré́ & Barra Bonita & Tatuí & 27,8 & 28,4 \\
\hline Caçapava & Taubaté & Santa Branca & S. J osé dos Campos & São Bento do Sapucaí & Salesópolis & Aparecida & 8,4 & 6,4 \\
\hline Cubatão 2 & Santo André & Cubatão 1 & Mauá & S. Bernardo do Campo & Mogi das Cruzes & Biritiba Mirim & 6,9 & 6,0 \\
\hline Dourado & Araraquara & Boraceia & Barra Bonita & Santa M. Serra & Itajú & Matão & 9,7 & 8,6 \\
\hline Guarujá & Cubatão & Santo André & S. Bernardo do Campo & Mauá & Mogi das Cruzes & Biritiba Mirim & 13,9 & 13,1 \\
\hline Itanhaém & Itariri & J uquitiba & Itapecerica da Serra & Cubatão & S. Bernardo do Campo & Santo André & 5,5 & 3,8 \\
\hline Itaporanga & Itararé & Timburí & Pirajú & Buri & Águas Santa Bárbara & Apiaí & 26,7 & 23,1 \\
\hline Itirapina 2 & Itirapina 1 & Piracicaba & Leme & São Carlos & Mogi Guaçu & Cosmópolis & 12,9 & 9,1 \\
\hline Lins & J úlio Mesquita & Reginópolis & Barbosa & Queirós & Mundo Novo (Graça) & I bitinga & 41,0 & 37,0 \\
\hline Martinópolis & Rancharia & Pres. Bernardes & Lutécia & Queirós & Guararapes & Barbosa & 23,6 & 20,2 \\
\hline Paranapanema & Itatinga & Buri & Guaré́ & Botucatu 1 & Pirajú & Tatuí & 4,4 & 5,2 \\
\hline PedroToledo & J uquitiba & Itariri & Itapecerica da Serra & Cotia & Tapiraí & Ibiúna & 20,2 & 13,4 \\
\hline Piacatu & Guararapes & Queirós & Rancharia & Lavínia & Barbosa & Lutécia & 15,3 & 13,0 \\
\hline
\end{tabular}

IQD = Inverso do Quadrado da Distância; Krig.=krigagem; Botucatu 1=Estação Botucatu; Botucatu 2 =Estação Bairro Anhúmas; Cubatão 1= Estação Piaçaguera; Cubatão 2 = Estação Terceiro Plano da Serra Nova; Itirapina 1 = Estação Visconde de Rio Claro; Itirapina 2 =Estação Graúna; a distância entre as estações e a respectiva cidade aumenta no sentido de 1 para 6. 
das mesmas observações anteriores, foram encontrados erros máximos altos, em especial para tempos de retorno mais baixos, chegando-se próximo a $80 \%$, para tempos de duração acima de $1.000 \mathrm{~min}$, para ambos os métodos, e tempo de retorno de cinco anos. Contudo, dificilmente estes valores são aplicados a projetos em pequenas bacias.

Para a estação deParanapanema, o comportamento dos erros mostrou-se semel hante ao das estações anteriores, constatando-se, no entanto, erros consideravel menteinferiores, verificando-se mel hor desempenho da krigagem para tempos de retorno inferiores a 25 anos e tempos de duração inferiores a 1.000 min e, para tempos de duração entre 100 e $1.000 \mathrm{~min}$, para todos os tempos deretorno. Os erros máximos encontrados ficaram em torno de $9 \%$ para ambos os métodos, porém para a krigagem isto ocorreu para tempos de duração muito altos.

$\mathrm{Na}$ figura 3, podem-se avaliar os erros proporcionados pel os métodos de interpolação para as estações de Cubatão e I taporanga. Para ambas as local idades, a krigagem proporcionou os menores erros médios (Quadro 2). No entanto, para a cidade del taporanga, foram constatadas mai ores diferenças entre os métodos para tempos de duração da precipitação inferiores a $100 \mathrm{~min}$, especialmente para tempos de retorno menores que 25 anos. A partir deste ponto, pode-se observar que os métodos praticamente se igualam, com ambos gerando maiores erros. Os erros máximos encontrados ficaram próximos a $50 \%$, para ambos os métodos, em qual quer tempo de retorno. Para Cubatão, observase comportamento semel hante ao de I taporanga. No entanto, para os tempos de retornoacima de 25 anos, percebe-se mel hora proporcionada pela interpolação via krigagem, observando-se diferenças bem superiores àquelas encontradas para I taporanga.

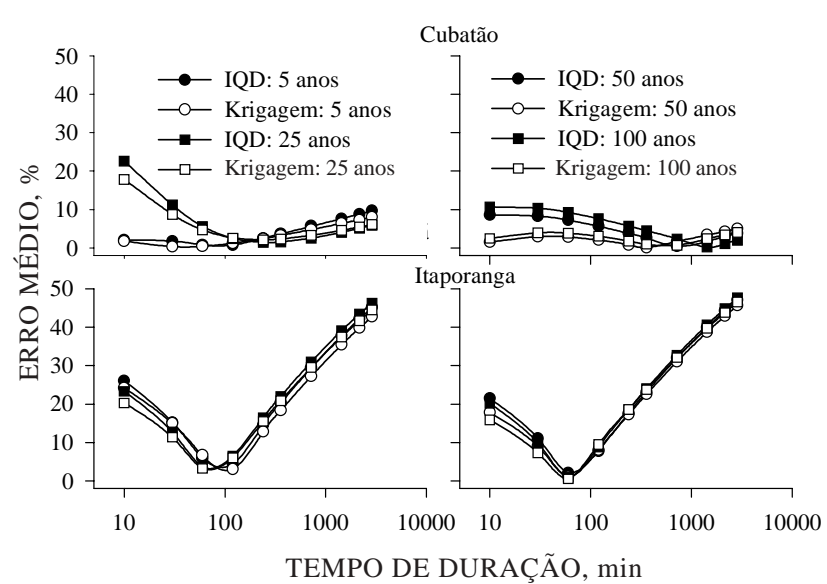

Figura 3. Relação entre erro das interpolações dos parâmetros de equações de chuvas intensas por krigagem e IQD com tempos de duração e de retorno para as localidades de Cubatão e Itaporanga.
A figura 4 apresenta o comportamento dos erros para as estações das cidades de I tirapina eCaçapava. Observa-se semel hança entre os erros para ambas as localidades, com desempenho superior da krigagem para tempos de duração da precipitação superiores a $100 \mathrm{~min}$, para I tirapina, esuperiores a 60 min para Caçapava. Nesta situação, a diferença proporcionada pela krigagem foi consideravelmente superior, em especial para Itirapina, onde os erros máximos foram de cerca de $30 \%$, para o IQD, em todos os tempos de retorno, e, para a krigagem, este percentual apenas para os tempos 50 e 100 anos. J á para Caçapava, os val ores máximos foram de $35 \%$ para krigagem no tempo de retorno 100 anos e $22 \%$ para o IQD, também neste tempo de retorno. Contudo, verifica-sequeos erros proporcionados pela krigagem foram inferiores para tempos de duração acima de 60 min, o que, na média, resultou em erro inferior para esse método.

$\mathrm{Na}$ figura 5, são apresentados os erros para as estações de Dourado el tanhaém, que geraram duas situações distintas, apesar de ambas terem produzido um erro menor na krigagem em relação a IQD para todas as situações de tempo de duração e tempo de retorno. Na primeira, os erros diminuem com o aumento do tempo de duração em todos os tempos de retorno. À medida que, para um mesmo tempo de duração, aumenta-se o tempo de retorno, verifica-se um aumento dos erros. Para a estação de I tanhaém, verifica-se um ponto de mínimo erro em cada tempo deretorno. Notam-se, ainda, para as duas localidades, erros máximos semel hantes, em torno de $15 \%$, para ol QD, e $13 \%$, para krigagem; no entanto, para Itanhaém, os erros mínimos foram menores, proporcionando erro médio menor (Quadro 2).

Pela figura 6, pode-se verificar o comportamento dos erros para as demais estações testadas. Estas

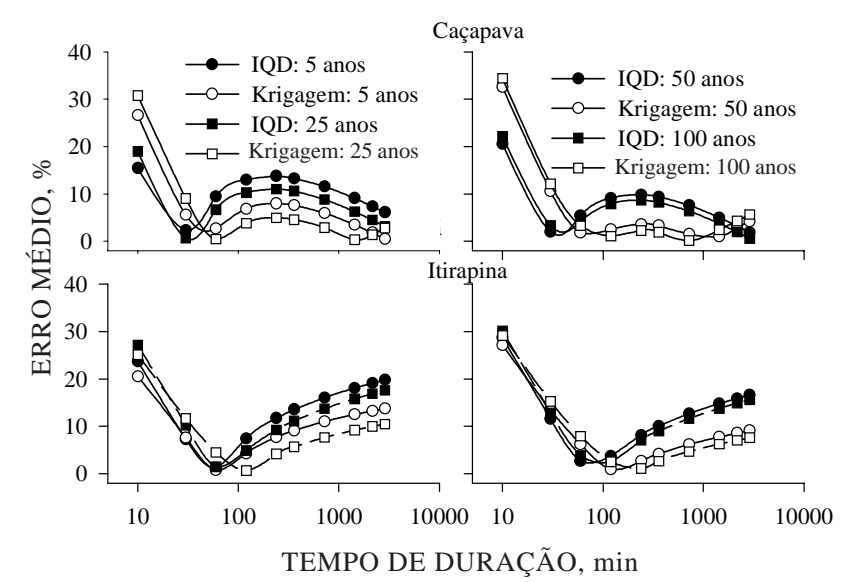

Figura 4. Relação entre erro das interpolações dos parâmetros de equações de chuvas intensas por krigagem e IQD com tempos de duração e de retorno para as localidades de Itirapina e Caçapava. 


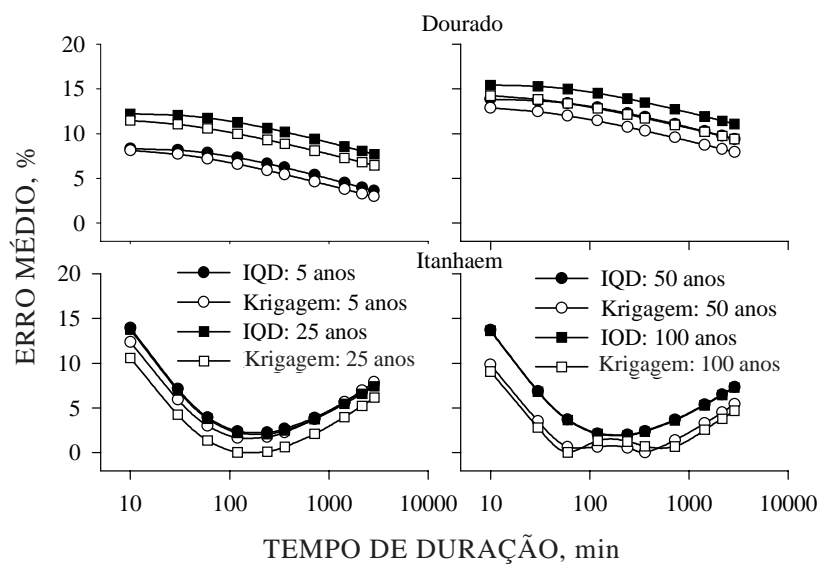

Figura 5. Relação entre erro das interpolações de parâmetros de equações de chuvas intensas por krigagem e IQD com tempos de duração e de retorno para as localidades de Dourado e Itanhaém.

estão compondo a mesma figura por terem apresentado, como características comuns: a krigagem sempre com os menores erros, independentemente de tempo de duração e tempo de retorno e tendência de aumento dos erros à medida que o tempo de duração aumenta. No entanto, para as estações de Lins, Martinópolis e Pedro de Toledo, observa-se o mel hor desempenho da krigagem à medida que aumenta o tempo de duração, aumentando as diferenças entre os métodos. Para as estações de Piacatu e Guarujá, o desempenho da krigagem ésuperior para os menores tempos de duração. Conforme já comentado com base apenas em erros médios, pode-se, por meio desta figura, visualizar o melhor desempenho da krigagem para as estações de Martinópol is e Pedro de Toledo, onde se observa uma diferença considerável de erros em todas as situações. Da mesma forma, para a estação de Lins, foram verificados erros médios muito superiores aos observados para as demais localidades, por ambos os métodos, em especial o IQD. A figura 6 revela ainda que os erros máximos ficaram em torno de $80 \%$, para IQD, e $70 \%$, para krigagem, muito superiores aos observados para as outras estações.

De maneira geral, com base nos resultados obtidos, o método de interpolação geoestatístico mostrou melhor desempenho, haja vista a predominância de menores erros produzidos por ele, mesmo em situações em que o inverso do quadrado da distância propiciou menores erros médios. Observou-se a predominância da krigagem, em especial para interval os detempo de retorno etempo de duração típicos de projetos hidráulicos em pequenas bacias. Com o advento de técnicas de programação computacional, pode-se superar facilmente o principal obstáculo práticoimposto pela krigagem, que é a necessidade de cálculos mais

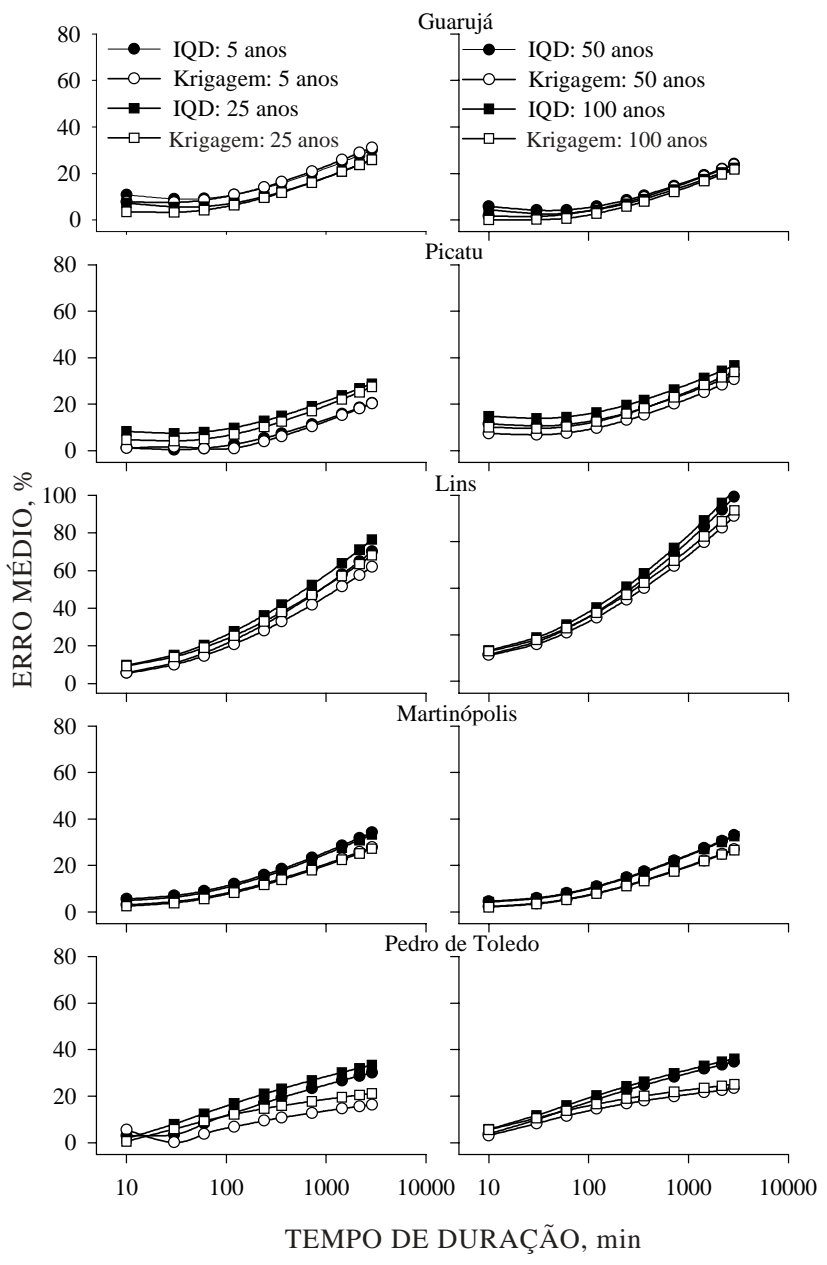

Figura 6. Relação entre erro das interpolações dos parâmetros de equações de chuvas intensas por krigagem e IQD com tempos de duração e de retorno para as localidades de Guarujá, Piacatu, Lins, Martinópolis e Pedro de Toledo.

complexos. Isto ajudará implementar programas computacionais de mapeamento da chuva intensa como aquel es baseados nos Sistemas de I nformação Geográfica, a fim de proporcionar espacialização destes parâmetros com maior exatidão, reduzindo substancialmente os erros de estimativa.

\section{CONCLUSÕES}

1. Os parâmetros da equação de chuvas intensas apresentaram dependência espacial, com bom ajuste dos model os exponencial ( $K, B$ e $C$ ) e esférico (a).

2. Embora ambos os métodos testados tenham apresentado baixos erros médios, a krigagem foi o método que propiciou os menores erros de interpolação dos parâmetros. 


\section{LITE RATURA CITADA}

ASSAD, M.L.L.; ASSAD, E.D. \& EVANGELHISTA, B.A. Chuvas extremas na região dos cerrados. In: ASSAD, C.D., coord. Chuva no cerrado: análise e espacialização. Brasília, Empresa Brasileira de Pesquisa Agropecuária, 1994. p.4954.

CARDOSO, C.O.; ULLMANN, M.N. \& BERTOL, I. Análise de chuvas intensas a partir da desagregação das chuvas diárias de Lages e de Campos Novos (SC). R. Bras. Ci. Solo, 22:131140, 1998.

CHOW, V.T.; MAIDMENT, D.R. \& MAYS, L.W. Appied hydrology. New York, McGraw-Hill, 1988. 572p.

DIRKS, K.N.; HAY, J .E.; STOW, C.D. \& HARRIS, D. Highresolution studies of rainfall on Norfolk Island Part II: Interpolation of rainfall data. J . Hydrol., 208:187-193, 1998.

FROEHLICH, D.C. Short-duration-rainfall intensity equations for drainage design. J . Irrig. Drainage Eng., 119:814-828, 1993.

GENOVEZ, A.B. Vazões máximas. In: PAIVA, J.B.D. \& PAIVA, E.M.C.D., orgs. Hidrologia aplicada à gestão de pequenas bacias hidrográficas. Porto Alegre, Associação Brasileira de Recursos Hídricos, 2001. p.33-112.

GOTWAY, C.A.; FERGUSON, R.B.; HERGERT, G.W. \& PETERSON, T.A. Comparison of Kriging and Inversedistance methods for mapping soil parameters. Soil Sci. Soc. Am. J ., 60:1237-1247, 1996.

HAAN, C.T. Statistical methods in hidrology. 2.ed. Ames, The I owa State University Press, 1979. 377p.

J OURNEL, A.G. Fundamentals of geostatistics in five lessons. Washington, American Geophysical Union, 1989. 40p.

J OURNEL, A.G. \& HUIJ BREGTS, Ch.J . Mining geostatistics. London, Academic Press, 1978. 600p.

LASLETT, G.M. Kriging and splines: an empirical comparison of their predictive perfomance in some applications. J . Am. Stat. Assoc., 89:391-400, 1994.
McBRATNEY, A.B. \& WEBSTER, R. Choosing functions for semivariograms of soil properties and fitting them to sampling estimates. J . Soil Sci., 37:617-639, 1986.

MELLO, C.R.; FERREIRA, D.F.; SILVA, A.M. \& LIMA, J .M. Análise de modelos matemáticos aplicados ao estudo de chuvas intensas. R. Bras. Ci. Solo, 25:693-698, 2001.

MYERS, D.E. Spatial interpolation: an overview. Geoderma, 62:17-28, 1994

PANNATIER, Y. "MS-WINDOWS Programs for exploratory variography and variogram modeling in 2D". In: CAPASSO, V.; GIRONE, G. \& POSA, D., eds. Statistic of spatial processes: theory and applications. Bari, Istituto per Ricerche de Mathematica Applicata (IRMA), 1994. p.165170.

PINTO, F.R.L. Equações de intensidade-duração-freqüência da precipitação para os estados do Rio de J aneiro e Espírito Santo: estimativa e espacialização. Viçosa, Universidade Federal de Viçosa, 1999. 70p. (Tese de Mestrado)

RIGHETTO, A.M. Hidrologia e recursos hídricos. São Carlos, Universidade de São Paulo, 1998. 840p.

SILVA, D.D.; VALVERDE, A.E.L.; PRUSKI, F.F. \& GONÇALVES, R.A.B. Estimativa e espacialização dos parâmetros da equação de intensidade-duração-frequencia da precipitação para o Estado de São Paulo. R. Eng. Agric., 7:70-87, 1999.

THOMPSON, S.K. Sampling. New York, Wiley-Interscience Publication, 1992. 343p.

TUCCI, C.E.M. Model os hidrológicos. PortoAlegre, Universidade Federal do Rio Grande do Sul, 1998. 669p.

VIEIRA, S.R. Geoestatística em estudos de variabilidadeespacial do solo. In: NOVAIS, R.F.; ALVAREZV., V.H.\& SCHAEFER, C.E.G.R., eds. Tópicos em ciência do solo. Viçosa, Sociedade Brasileira de Ciência do Solo, 2000. p.1-54.

VIEIRA, S.R.; LOMBARDI NETO, F. \& BURROWS, I.T. Mapeamento da chuva diária máxima provável para o estado de São Paulo. R. Bras. Ci. Solo, 15:93-98, 1991.

VOLTZ, M. \& WEBSTER, R. A comparison of kriging, cubic splines and classification for predicting soil properties from sample information. J. Soil Sci., 41:473-490, 1990. 
C.R. MELLO et al.

R. Bras. Ci. Solo, 27:925-933, 2003 